\title{
ORIGINAL
}

\section{NECESIDADES EN SALUD DE LA POBLACIÓN DESPLAZADA POR CONFLICTO ARMADO EN BOGOTÁ}

Amparo S Mogollón Pérez (1,2), M. ${ }^{a}$ Luisa Vázquez Navarrete (3) y M. ${ }^{a}$ del Mar García Gil (4)

(1) Universidad del Rosario, Colombia

(2) Universidad Autónoma de Barcelona

(3) CHC Consultoria i Gestió, SA, Barcelona

(4) Hospital Josep Trueta, Girona

\section{RESUMEN}

Fundamentos: El desplazamiento de poblaciones como consecuencia de un conflicto armado, conlleva generalmente un deterioro en las condiciones de vida y de la salud de quienes lo padecen. El objetivo de éste artículo es analizar las necesidades en salud percibiobjetivo de éste artículo es analizar las necesidades en salud percibi-
das por hombres y mujeres desplazados por el conflicto armado y sus principales estrategias de resolución.

Métodos: Se realizó una investigación cualitativa mediante entrevistas individuales semi-estructuradas a 31 personas desplazadas en fase de transición. Se hizo un análisis narrativo de contenido segmentando la información por edad y sexo. El área de estudio estaba constituida por cinco localidades de la ciudad de Bogotá.

Resultados: El compromiso de la salud mental y de la estabilidad psicosocial, los problemas con la alimentación y en menor proporción las afecciones gastrointestinales y respiratorias son los principales problemas de salud referidos por ambos grupos de informantes. La dificultad de acceso a los servicios de salud emerge como problema adicional La precaria situación económica subyace a los problemas y a las los problemas de salud, además de los servicios de salud, las personas desplazadas emplean otras estrategias a su alcance. Las necesidades expresadas son coherentes con los problemas percibidos. Se observan algunas diferencias en la apreciación de los problemas y en las estrategias de solución, entre hombres y mujeres y entre grupos de edad.

Conclusión: En el ámbito de la salud, se requieren acciones que permitan mejorar el acceso a los servicios, así como estrategias específicas para la rehabilitación psicosocial de la población desplazada que tengan en cuenta las diferencias en el interior del colectivo.

Palabras claves: Necesidades y demandas en servicios de salud. Campos de refugiados. Estudio cualitativo. Colombia.

\section{ABSTRACT}

\section{Health-Related Needs of the Displaced Population Due to Armed Conflict in Bogota}

Background: The displacement of populations as the result of an armed conflict generally entails a worsening of the living and health conditions of those undergoing such a displacement. This paper is aimed at analyzing the health-related needs perceived by men and women displaced by the armed conflict and their main strategies to address those needs.

Methods: A qualitative study was carried out by means of semistructured individual interviews to 31 displaced men and women in the transition stage. A narrative analysis of the contents was conducted, segmenting the information by age and sex. The area under study was made up of five localities in the city of Bogotá.

Results: The negative effects on their mental health and psychosocial stability, access to food and, to a lesser degree, gastrointestinal and respiratory disorders, are the main health problems reported by both groups of informants. The difficulty of accessing health care services comes up as an added problem. The precarious economic situation underlies the health care-related needs and problems. To solve their health problems, in addition to the health care services, they employ other strategies within their reach. Expressed needs are coherent with the problems perceived. Some differences between women and men and age groups were observed concerning the definition of the problems and employed strategies.

Conclusion: In the health field, actions are required in order to improve their access to services in addition to specific strategies for the psychosocial rehabilitation of the displaced population which take into account the differences existing within this group.

Key words: Health services needs and demand. Refugee camps. Qualitative study. Colombia.
Correspondencia:

Amparo Susana Mogollón Pérez

Universidad de Rosario

Carrera, 24 63C-69 Bogotá

Colombia

Correo electrónico: susana_mogollon@hotmail.com

\section{INTRODUCCIÓN}

El conflicto armado, una de las principales manifestaciones de la violencia en Colombia, no sólo ha provocado el enfrentamiento entre los grupos armados y las fuer- 
zas del Estado, sino que ha llegado a afectar de manera directa a la población civil ${ }^{1,2}$. Como alternativa de supervivencia, la población se desplaza a las áreas urbanas, siendo Bogotá el principal centro receptor del país ${ }^{3}$. Sólo entre agosto de 1998 y el primer trimestre de 2002 fueron desplazadas 1.091 .320 personas (un promedio de mil personas diarias) y los desplazamientos masivos aumentaron de manera significativa ${ }^{4}$.

La calidad de vida de las personas desplazadas tiende a empeorar en la ciudad, especialmente en la fase de transición, que es el periodo transcurrido entre la elección de un asentamiento temporal hasta que logran su reubicación definitiva. En esta etapa cesan las ayudas humanitarias de emergencia y es común que se deteriore su situación económica, tornándose el asentamiento cada vez mas difícil ${ }^{5,6}$. En la mayoría de los casos, los desplazados se ubican en áreas cuyas condiciones sanitarias y de pobreza favorecen el deterioro de la salud ${ }^{7,8}$. Los estudios que tratan de evaluar los problemas de salud que afectan a las personas desplazadas en Colombia han arrojado resultados diversos. Algunos reportan problemas con características epidemiológicas ligadas a la pobreza (infección respiratoria aguda, enfermedad diarreica aguda, enfermedades de la piel y parasitosis intestinal $)^{9,10}$, en tanto que otros, destacan el dolor general, las afecciones cardiovasculares, dermatológicas, respiratorias, digestivas y, en menor proporción, las enfermedades de tipo psicosomático ${ }^{11}$. A esta problemática se añade la dificultad de acceder a los servicios de salud. Aunque el Estado colombiano creó el Sistema Nacional de Atención Integral a la Población Desplazada por la Violencia, que une esfuerzos de diferentes instituciones y sectores, la oferta de servicios de salud resulta insuficiente para atender sus necesidades ${ }^{12}$.

Los estudios existentes sobre los problemas de salud derivados del desplazamiento, además de proporcionar resultados discrepantes se han centrado fundamentalmente en el perfil epidemiológico y los costos para el sistema de salud desde un punto de vista normativo y cuantitativo.

El objetivo de este estudio es identificar los problemas de salud y las necesidades de atención a la salud que son percibidos por los hombres y mujeres desplazados por conflicto armado, así como las estrategias que utilizan en la búsqueda de la salud. Se circunscribe a las personas desplazadas en fase de transición, por su mayor vulnerabilidad, y a la ciudad de Bogotá, por ser el mayor centro receptor de población desplazada del país.

\section{SUJETOS Y MÉTODOS}

Se realizó un estudio descriptivo exploratorio, cuya metodología pertenece al enfoque cualitativo de la investigación social. El trabajo de campo se desarrolló en los meses de marzo y abril de 2002. El área de estudio fue Bogotá, específicamente las localidades de Bosa, Ciudad Bolívar, Engativá, Tunjuelito y Usme.

Se utilizó la entrevista individual semiestructurada, es decir, con una guía de temas, que no constituye un cuestionario y que permite al investigador abordar todas las áreas de interés, sin seguir un orden, ni una formulación preestablecida. Se trataron los siguientes temas: cambios observados en la salud posterior al desplazamiento, principales problemas de salud, causas de los problemas, necesidades de atención en salud, acceso a los servicios de salud y mecanismos de solución para los problemas de salud. La duración de las entrevistas fue de 60 a 90 minutos. En los 6 casos en que se consideró necesario profundizar en algunos temas, se realizó una segunda visita domiciliaria. Todas las entrevistas fueron grabadas y posteriormente transcritas textualmente.

Muestra. Se hizo una selección intencional de los informantes, buscando reflejar las condiciones de los adultos desplazados en la ciu- 
dad de Bogotá y recoger el máximo posible de variedad en sus opiniones ${ }^{13,14}$. Los criterios utilizados para la selección de los entrevistados fueron: ambos sexos, edad superior a 18 años, en fase de transición en el desplazamiento y haber elegido Bogotá como lugar de asentamiento. Participaron 31 desplazados, 18 mujeres y 13 hombres, entre los 18 y 79 años de edad. Se identificó a los informantes a partir de la base de datos de una organización receptora de personas desplazadas que llegan a la ciudad. Se les contactó telefónicamente, se solicitó su consentimiento para participar en el estudio y se acordaron horarios y días para la realización de las entrevistas en sus lugares de residencia.

Análisis de la información. Se realizó un análisis narrativo del contenido. Los datos fueron segmentados de manera manual, según sexo y edad de los entrevistados, en hombres y mujeres y éstos, a su vez, en adultos jóvenes (18 a 44 años) y adultos mayores (45 a 79 años). Se leyeron las transcripciones y se desarrollaron las categorías de análisis de forma mixta: a partir de los guiones utilizados y de los temas emergentes de las entrevistas. Mediante anotaciones en el margen de las entrevistas se estableció un código descriptivo que atribuía un contenido a cada segmento de texto señalado. Se agruparon los contenidos de cada entrevista que hacían referencia a las categorías desarrolladas, estableciendo un patrón de datos. Posteriormente se crearon subcategorías que resultaron de la agrupación de las respuestas similares. En una segunda etapa del análisis se clasificó la información por grupos de informantes, para establecer semejanzas y diferencias.

Calidad de los datos. Para garantizar la calidad de los datos en las entrevistas participaron una entrevistadora y una observadora. La observadora tomaba notas durante la entrevista. Las transcripciones fueron textuales, completadas con las notas de la observadora y verificadas con la persona entrevistada. El análisis lo realizaron dos investigadoras. Los resultados se contrastaron con los obtenidos de otras fuentes de información bibliográfica.

\section{RESULTADOS}

\section{Problemas de salud y sus causas}

Del análisis del discurso de las personas entrevistadas se identificaron dos tipos fundamentales de problemas de salud, los derivados del desplazamiento y aquellos no relacionados con éste.

a) Problemas de salud derivados del desplazamiento

Las personas entrevistadas, tanto hombres como mujeres, refieren tres tipos de problemas de salud como consecuencia directa del desplazamiento: el compromiso de la salud mental y la pérdida de la estabilidad psicosocial, los relacionados con la alimentación y, en menor grado, dolores, problemas gastrointestinales y respiratorios. Las principales diferencias entre mujeres y hombres se observan en el reconocimiento del impacto psicosocial del desplazamiento a nivel individual y familiar.

Compromiso de la salud mental. La gran mayoría de hombres y mujeres coinciden en que han sufrido situaciones de «estrés», «tensión», «preocupación»o «intranquilidad», que los ha afectado tanto a sí mismos como a su familia (un estrés pero terrible, no hay tiempo para caricias, no hay tiempo para nada. Se vive las 24 horas al día en el problema...mi señora anda con los pelos de punta, yo vivo neurótico, hombre mayor). También refieren cambios en el estado de ánimo. Los jóvenes manifiestan tristeza por las situaciones que han tenido que vivir, expresando sentimientos de frustración e impotencia (uno se pone triste......hay veces me pongo a pensar ¿por qué la vida es así?, ¿por qué nos ha tocado ésta situación? y 
además no puedo hacer nada por cambiarla, hombre joven) o, por el contrario, actitudes intolerantes hacia otros miembros de la familia (de pronto los niños me hablan y a mi me ofende que me hablen, y yo entiendo que eso no es normal, mujer joven). Para las personas mayores, la experiencia sufrida y la pérdida de sus familiares les lleva a no querer seguir viviendo (lamentablemente es un dolor que queda. Eso nunca lo sanea uno tal vez ni con la muerte. Para mí hubiera sido preferible que me hubieran quitado la vida, que hubieran acabado conmigo y no con mis hijos, mujer mayor). Finalmente, tanto hombres como mujeres, refieren trastornos del sueño, que describen como dificultad para conciliarlo o como pesadillas al recordar hechos violentos.

Problemas psicosociales. La violencia intrafamiliar y las dificultades de adaptación surgieron como otro grupo de problemas provocado por el desplazamiento y estrechamente relacionado con el anterior. Se observaron diferencias de apreciación en las respuestas de hombres y mujeres. Una gran parte de las mujeres, principalmente las jóvenes, refieren cambios en su comportamiento que las lleva a replicar acciones violentas o a usar la violencia como mecanismo de corrección de sus hijos (a raíz de eso yo he cambiado mucho, por que yo, todo es a la violencia, yo todo tengo que arreglarlo es a los problemas, a los golpes, mujer joven). Los hombres, por el contrario, reconocen esta problemática de manera limitada. Sólo uno hizo referencia a esta situación y como conducta observada en otros desplazados (entre hermanos, entre padres, hijos, hay conflictos graves incluso que a veces hay amenazas de muerte, ataque físico, maltrato marido- mujer, maltrato padres -hijos, hombre mayor). Otros problemas psicosociales descritos se refieren a la adaptación a la ciudad. Los hombres mayores expresan la dificultad en el cambio de tipo de trabajo ( $a \mathrm{mi}$ sinceramente me daba pena trabajar con una carretilla en la calle, hombre mayor). A su vez, las mujeres refieren preocupación por los riesgos que no tenían en sus lugares de origen y que pueden afectar a la familia (aquí hay mucha pandilla, entonces, el chino; a mí me da afán que haga amistad por ahí, y eso me da mucho miedo a mi, o que coja las drogas o algo así, mujer joven).

Problemas con la alimentación. La mayoría de los entrevistados describe las dificultades relacionadas con la alimentación como uno de los problemas más importantes y lo asocian a la pérdida de peso y a una mayor susceptibilidad para adquirir enfermedades (poco consumía o sea dietéticamente me desnutrí mucho y llegué como un fantasma o sea recién ahora estoy engordando, hombre mayor). Algunas mujeres jóvenes, atribuyen a la baja ingesta alimenticia sus dificultades con la lactancia (cuando no hay mucho líquido para tomar de noche hum...tremendo, porque no me sale nada de leche y la niña llora y llora.. Y con la angustia mas se me seca y que hago Dios mío?, mujer joven).

Otros problemas de salud. Aunque con menor frecuencia, los entrevistados manifestaron otros problemas de salud consecuencia del desplazamiento: problemas gastrointestinales (la gastritis a mi molesta, yo creo que es la preocupación, hombre mayor); (hemos estado susceptibles a más enfermedades de tipo digestivo, el cambio de agua, el cambio de ubicación ha sido muy nefasto...al principio vivíamos con diarreas, hombre mayor), dolores de cabeza y problemas respiratorios.

\section{b) Problemas de salud no derivados por el desplazamiento}

Aparte de los problemas de salud que relacionan de manera directa con el desplazamiento, las personas entrevistadas indicaron otros problemas de salud: dolores, problemas cardiovasculares, odontológicos, dermatológicos, urinarios y de visión. Entrevistados de los diferentes grupos refirieron la presencia de dolor en diversas partes del 
сиеrpo. Las alteraciones a nivel cardiovascular fueron enunciadas de manera exclusiva por hombres y mujeres mayores como hipertensión o fallas de circulación. Finalmente, en el discurso de las mujeres, tanto jóvenes como mayores, son frecuentes las descripciones de trastornos ginecológicos como trastornos menstruales y dolor en los ovarios.

\section{c) Causas de los problemas de salud}

Gran parte de las personas entrevistadas coincide en la identificación de las causas de sus problemas de salud. En primer lugar, hombres y mujeres señalan las dificultades económicas que les imposibilita la satisfacción de sus necesidades básicas. Algunos también atribuyen la ansiedad y el estrés que sufren a la violencia que han visto o padecido (yo veía que pasaban, hacían atentados, pues que tumbaban pueblos y eso me sentía muy mal, entonces creo que eso me afectó emocionalmente, hombre joven). Finalmente, algunos entrevistados consideran las nuevas condiciones medioambientales y climáticas, a que se ven sometidos por el desplazamiento, como causas de los problemas infecciosos y respiratorios (tenemos el río aquí muy cerca, que es un río muy contaminado, puede haber mucha enfermedad, muchas epidemias, por basuras y tanto pantano y zancudos, mujer mayor).

Dificultades en el acceso a los servicios de salud

En el discurso de hombres y mujeres aparece de manera repetida la existencia de limitaciones para acceder a los servicios de salud y que atribuyen a diversos factores, tanto institucionales como individuales. En relación a los primeros, son muchos los que refieren no haber recibido atención en algunas instituciones que manifestaban insuficiencia en la infraestructura (no me recibieron porque no había camas y se supone que el desplazado tiene una prioridad para la atención. Me enviaron para el Meissen y lo mismo no habían camas, mujer joven). Así mismo, perciben actitudes discriminatorias generadas por su actual condición (esa carta, es lo más discriminatorio; porque en algunos hospitales nos tienen catalogados como indigentes, mujer mayor); (Yo creo que es mejor tener sida y no ser desplazado, sí, porque en todas partes uno toca las puertas y no recibe atención de ninguno, hombre joven). Para otros, la burocracia en muchos de los trámites se convierte en un obstáculo para su atención (teníamos al día todos los papeles en los que constaba que éramos desplazados, pero no nos sirvió para nada, y no lo quisieron recibir, que hasta que la Red no enviara una carta directamente no lo podían recibir y no hicieron nada. Y lo tuvieron de hospital en hospital, mujer joven). Entre los factores relativos a la población, de nuevo aparecen las dificultades económicas como la principal barrera existente. En muchas ocasiones no cuentan con recursos para el transporte, para la compra de los medicamentos que no les proporcionan las instituciones o para el pago de consultas cuando no han efectuado los trámites necesarios para acceder a los beneficios del Sistema General de Seguridad Social en Salud. Muchas de las mujeres que han asumido la jefatura del hogar no acuden a los servicios de salud por temor a perder su empleo (yo apenas llevo tres meses de estar trabajando ahí, y de pronto yo saco un día y al otro día me dicen: - lo que usted no quiere es trabajar, si no quiere trabajar pues no trabaje- y pues yo lo necesito, y para yo perder ese trabajo, es muy dificil volverlo a encontrar, mujer joven), en tanto que para otras, el ser cuidadoras de sus hijos o de terceras personas, se convierten en obstáculos para acceder a los servicios de salud (no he tenido los niños con quien dejarlos y siempre los hospitales quedan retirados, mujer joven). Por último, otra barrera para el acceso es la falta de información sobre los servicios que ofrecen las instituciones de salud y de los beneficios que ofrece el gobierno para la población desplazada. 


\section{Estrategias de solución para los problemas en salud}

En la búsqueda de solución para sus problemas y necesidades en salud muchos de los entrevistados, recurren a su entorno, básicamente instituciones de salud y redes sociales o familiares (iglesia, amigos, familia, vecinos, etc). Otros utilizan estrategias a nivel interno, a partir del reconocimiento de sus propias capacidades. Dentro de las estrategias externas, existió coincidencia en la respuesta de la mayor parte de los entrevistados al destacar la asistencia a los servicios de salud y el cumplimiento, en la medida de sus posibilidades, de los tratamientos prescritos (primero que todo ir al médico, visitar al médico y pues hacerme el tratamiento, que el médico me dé, mujer joven). Fueron muy pocos los entrevistados que manifestaron no tener interés en asistir a las instituciones de salud. La búsqueda de ayuda en redes sociales y familiares es una alternativa frecuente de la mayoría de las mujeres, y algunos hombres jóvenes, destacando el apoyo tanto asistencial como social de organizaciones no gubernamentales y de la iglesia. En cuanto a las alternativas internas, hombres y mujeres refirieron prácticas personales, como la oración o intentar olvidar los recuerdos negativos, para encontrar un «alivio» o serenidad frente a su situación (todos esos dolores se los dejo a mi Diosito y eso me van pasando, hombre joven); (Yo oro y siento que me tranquilizo, mujer mayor). También manifestaron, principalmente las mujeres, el uso de tratamientos caseros para el manejo de las afecciones consideradas como «leves» (dolores de cabeza, estomacales y cuadros de diarrea, entre otros), tanto para sí mismas como para su familia (cuando ellos tiene un dolor de estomago, yo les preparo hierbas, pues es lo único que uno sabe hacer así en la casa, mujer joven).

\section{Necesidades de atención en salud}

Además de un acceso garantizado a los servicios de salud, la mayor parte de los entrevistados, excepto los hombres mayores, expresaron la necesidad de recibir apoyo psicológico para la resolución de sus propios conflictos (falta también como de un psicólogo, en verdad una persona que le de todas esas atenciones que uno en verdad necesita... porque hay muchas cosas que decir, hombre joven). Las mujeres jóvenes consideraban el apoyo psicológico fundamental para el manejo de los problemas de otros miembros de su familia (de pronto él le cuente sus cosas y le ayude, de pronto el se pueda desahogar con otro mejor que conmigo, mujer joven). Muchos entrevistados manifestaron la necesidad de recibir medicamentos que no siempre les son proporcionados por las instituciones como establece la ley (que los médicos formulen los medicamentos que estén en el stock, porque va uno a pedirlos y no están, que espere. y uno con la fórmula no mas no se alienta, hombre joven). Tanto hombres como mujeres expresaron la necesidad de contar con apoyo nutricional para toda la familia (lo que pediría es nutrición para mí y los niños, por lo que estamos tan mal de alimentos, mujer joven). Sólo unos hombres jóvenes hicieron referencia a la orientación nutricional como una estrategia para modificar sus hábitos alimenticios. En menor proporción se enunció la asistencia a medicina general y odontología, el acceso a programas preventivos, elementos protésicos y servicios de rehabilitación.

\section{DISCUSIÓN}

En los discursos analizados se identifican dos tipos fundamentales de problemas de salud, los derivados del propio desplazamiento, más frecuentes y detalladamente mencionados, y otros no directamente relacionados con la situación de desplazados y que se corresponden con el perfil epidemiológico de la población colombiana ${ }^{15}$. En relación a los problemas originados por el desplazamiento, las personas entrevistadas refieren en primer lugar compromiso de la 
salud mental y alteraciones psicosociales, seguido de los relativos a cambios en la alimentación y, en menor grado, dolores, problemas gastrointestinales y respiratorios. Estos dos aspectos, la diferenciación de los problemas según su relación con el desplazamiento y la importancia atribuida en los discursos al compromiso de la salud mental y sus consecuencias psicosociales, como uno de los principales problemas de salud que evidencian, distingue estos resultados de otros estudios efectuados en Colombia. En éstos se suele encontrar la descripción general de la problemática de salud de los desplazados sin diferenciar su origen en el desplazamiento y, asimismo, el compromiso de la salud mental se reporta como un problema de menor magnitud ${ }^{16}$. No obstante, hay que destacar que tanto unos como otros son percibidos por los informantes como problemas de salud que comprometen su calidad de vida y requieren atención desde los servicios de salud. Pero, al mismo tiempo, estos resultados plantean la necesidad de explorarlos en mayor profundidad, para brindar una respuesta de los servicios de salud más adaptada a las distintas fases del desplazamiento.

Numerosos estudios, si bien la mayoría en población refugiada, han evaluado el impacto de la guerra sobre diferentes colectivos ${ }^{17} \mathrm{y}$ han analizado la magnitud del compromiso de la salud mental de la población afectada y los factores asociados ${ }^{18,29}$. Alrededor de un $40 \%$ de las personas expuestas a la violencia de la guerra presentan algún tipo de sintomatología psiquiátrica ${ }^{19}$. Aunque en Colombia se trate de desplazados y no refugiados, los resultados apuntan a que la baja representación de los problemas de salud mental en los estudios realizados pueda deberse al tipo de aproximación metodológica efectuada, en relación a instrumentos que no permitan la identificación de este tipo de problemas ${ }^{24} \mathrm{o}$ a un diferente enfoque en el análisis.

La salud mental no sólo parece estar comprometida como consecuencia de haber vivido el conflicto bélico, sino también por el rechazo y la estigmatización que genera en los desplazados su nueva condición social ${ }^{20}$. Se constituye así en uno de los problemas más importantes que padece la población desplazada. Los cambios de vida introducidos por el desplazamiento, junto con el compromiso mental de sus miembros, repercuten de manera directa en la estabilidad psicosocial de la familia. En muchos casos, la tensa situación por la que atraviesan favorece una dinámica de violencia a nivel intrafamiliar que se convierte en una prolongación y complejización del conflicto armado y del desplazamiento $^{21}$. En este aspecto, se observan diferentes posiciones en los hombres y las mujeres. La mujer, principalmente la joven, reconoce sus cambios actitudinales hacia los miembros de su familia y sus modificaciones en la comunicación, que pasa de estar basada en el afecto a estar basada en el poder $^{22,23}$. Por el contrario al hombre, aunque pueda identificar esta problemática en otros, parece que le sea más difícil reconocerse como agresor de su propia familia. Las diferencias parecen sugerir una visión del rol de género en la que el hombre detenta la autoridad y en la que el uso de la fuerza es un medio de ejercerla ${ }^{21} \mathrm{y}$, por tanto, no se percibe como problema. La percepción de su rol de género también podría explicar su mayor dificultad en adaptarse a un nuevo entorno laboral y cambios intrafamiliares.

En relación con el acceso a los servicios de salud, el colectivo estudiado percibe situaciones de exclusión y marginación. La dificultad para ser atendidos, puede ser un reflejo del problema que atraviesan los servicios de salud públicos en la actualidad, con un aumento de la demanda de atención y, al mismo tiempo, una disminución del presupuesto asignado ${ }^{24}$. No obstante, las dificultades burocráticas referidas también pudieran ser un indicio de una pobre comunicación interinstitucional en torno a las políticas existentes para la atención a la población desplazada. Los problemas de acceso, sin embargo, también se localizan en los propios desplazados, siendo muchos los factores que 
inciden en ello, pero destacándose como el más importante su precaria situación económica $^{23,8}$. En el caso de la mujer, como cabeza de hogar o como cuidadora, la decisión de asistir a los servicios de salud se torna particularmente difícil. Con frecuencia carece de medios para dejar a los menores a su cargo o debe escoger entre la realización de actividades que suponen un ingreso económico y la solución de sus propios problemas de salud. Otros de los factores que también limitan el acceso por parte de la población desplazada lo constituyen el desconocimiento de sus derechos y de los procedimientos a seguir.

La precariedad en sus ingresos no sólo condiciona el acceso a los servicios de salud y a los tratamientos prescritos, sino también a una vivienda adecuada y a una alimentación balanceada que cubra los requerimientos nutricionales de la familia. Esta situación, que puede llevar incluso a la mendicidad, está relacionada con la pérdida de sus bienes de subsistencia y a la dificultad para insertarse en la vida laboral. Muchos de los desplazados son de origen campesino, poseen un bajo nivel educativo ${ }^{5,8} \mathrm{y}$, por desconocer otros oficios, identifican pocas opciones para obtener ingresos. En este aspecto, existen diferencias entre hombres y mujeres. La mujer tiende a vincularse a trabajos domésticos y al sector informal, mientras que los hombres tienden a quedar desempleados, aunque algunos, principalmente jóvenes, se vinculan a labores de construcción, seguridad y a la economía informal ${ }^{23}$. Como consecuencia, la mujer se convierte en muchos casos en la proveedora económica del $\operatorname{hogar}^{8,23}$. Esta difícil situación económica es el elemento de mayor preocupación de hombres y mujeres y permea toda el discurso relacionado con la salud, tanto sobre los problemas percibidos, como sobre las estrategias de solución.

Las dificultades para adquirir una alimentación cuya calidad y cantidad les permita un adecuado balance nutricional es otro problema manifestado por los entrevistados y ya puesto de manifiesto por numerosos estudios en población desplazada ${ }^{6,8,11}$, así como su relación directa con el tiempo de asentamiento. Cuanto más tiempo transcurre desde que se asentó, mayor es la dificultad de los desplazados para la adquisición de alimentos, debido al cese de muchas de las ayudas humanitarias de emergencia y la dificultad para su inserción laboral ${ }^{6}$. La alimentación como necesidad básica a satisfacer es objeto de estrategias diseñadas por la población desplazada ${ }^{24}$, principalmente en las fases tempranas del desplazamiento. Sin embargo, su relación con las fases del desplazamiento sugiere que las acciones no deben limitarse a las ayudas nutricionales de emergencia, sino que se deberían involucrar mecanismos que posibiliten la seguridad alimentaria a largo plazo $^{25}$.

Las necesidades de atención a la salud descritas por los entrevistados se corresponden con los problemas percibidos: acceso a los servicios de salud y la atención en áreas específicas, como el apoyo psicológico y el soporte nutricional. La población desplazada conoce y utiliza los servicios de salud, pero también busca soluciones a sus problemas en otros ámbitos, como la religión. Además del limitado acceso a los servicios, esta conducta parece reforzar la idea de que los servicios de salud no están proporcionando la atención específica que necesita la población desplazada. Se plantea una reflexión ineludible, tanto para los servicios de salud como para los planificadores, en términos de diseñar estrategias que garanticen el acceso de los desplazados a los servicios de salud en un entorno de recursos limitados. En este sentido, sería importante adaptar las estrategias a las necesidades de cada fase y a las diferencias en los colectivos.

En este estudio destaca el apoyo psicológico como una de las principales necesidades de atención, dada la problemática de salud mental que tiene esta población y su propia percepción del problema. Sin embargo, los resultados sugieren una mayor capa- 
cidad de adaptación a la nueva forma de vida de los jóvenes frente a los mayores y de las mujeres frente a los hombres ${ }^{26}$. Por tanto, las estrategias en éste área, deberían partir del reconocimiento de las características y necesidades particulares de los distintos grupos objeto de la intervención. Sería necesario fortalecer y dar continuidad a los programas de intervención psicosocial elaborados por organismos nacionales e internacionales ${ }^{24,27}$, con el fin de que no actúen de manera aislada y discontinua, sino que hagan parte de una acción conjunta entre instituciones y colectivos, a largo plazo.

Finalmente, la complejidad de las causas subyacentes a los problemas de salud de las personas desplazadas señala la necesidad de una amplia política intersectorial que permita la reubicación o el retorno del sujeto y su familia, en condiciones adecuadas, como requisito fundamental para el logro de su desarrollo humano.

\section{AGRADECIMIENTOS}

A las mujeres y hombres desplazados que aceptaron compartir su problemática con las investigadoras. A la Facultad de Rehabilitación y Desarrollo Humano de la Universidad del Rosario (Colombia) y al Centro de Atención al Migrante de la ciudad de Bogotá por el apoyo brindado para la realización del estudio.

\section{BIBLIOGRAFÍA}

1. Sánchez G. La violencia in Colombia: new research, new questions. HAHR 1985; 65 (4):789-807.

2. Comittee for Refugees (US). Inmigration and Refugee Services of America. Colombia's Silent Crisis One million displaced by violence;1998:144.

3. Arquidiócesis de Bogotá, Codhes. Senderos Rostros Invisibles del desplazamiento en Bogotá. Bogotá: La Arquidiócesis; 2001.
4. Consultoría para los Derechos Humanos y el Desplazamiento. Aspectos críticos de la política pública de atención a la población desplazada 1998 2002. [citado 23 de Ago 2002]. Disponible en: www.codhes.org.co.

5. García M. Los desplazados por la violencia en Colombia. Con su dolor sin rumbo. Universitas Humanística 1999; XXVII (47): 15-31.

6. Programa Mundial de Alimentos Colombia. Estudio de Caso de las Necesidades Alimentarias de la Población Desplazada de Colombia. 2001 [citado 18 Nov 2001]. Disponible en: www.col.opsoms.org/desplazados/informes/pma/FinalReportSpanish htm

7. Toole M, Waldman R. Refugees and Displaced Persons. War Hunger and Public Health. JAMA 1993. 270(5): 600-605.

8. Procuraduría General de la Nación. Instituto de Estudios del Ministerio Público. Procuraduría Delegada para la Defensoría del Menor y la Familia. Raíces sin tierra Atención e impacto del Desplazamiento Forzoso. Santa Fe de Bogotá: La Procuraduría; 1999.

9. Morales M, Torres L. Principales problemas de salud de los desplazados por la violencia Pavarandó y Turbo. Programa de Urgencias, Emergencias y desastres. Medellín: Universidad de Antioquia Facultad Nacional de Salud Pública; 1998.

10. Gobernación del Valle del Cauca, Secretaria Departamental de Salud. «Hacia La Construcción de un Modelo Piloto para el diseño e Implementación de un Sistema de Información de Salud Pública y Vigilancia Epidemiológica en Poblaciones Desplazadas por Violencia Política» [citado 7 Feb $2002]$. Disponible en: www.Col.OpsOms.Org/Desplazados/Documentos/Sdsvalle/Creditos.Htm.

11. Organización Internacional para las Migraciones. Diagnóstico de población desplazada y comunidades de recepción en seis departamentos de Colombia [citado 20 nov 2001]. Disponible en: www.col.ops-oms.org/desplazados/informes/oim/ diagnosticooim.htm.

12. Muggah R. Capacidades Institucionales en medio del conflicto, Una evaluación de la respuesta en la Reubicación de la Población Desplazada en Colombia. Conflicto Armado: criminalidad, violencia y desplazamiento Forzado. Bogotá: Departamento Nacional de Planeación; 1999; XXX (3): 233-90.

13. Miles MB, Huberman AM. Qualitative data analysis. London; 1994. 
14. Taylor SJ, Bogdan R. Introducción a los métodos cualitativos de Investigación. Barcelona: Paidos; 1998.

15. Organización Panamericana de la Salud. Resúmenes Boletín Epidemiológico, La Salud en las Américas Perfiles básicos de salud de países en las Américas [citado 9 feb 2002]. Disponible en: www.paho.org/Spanish/SHA/prflCOL.htm\#morbimortal.

16. Secretariado Nacional de Pastoral Social. RUT informa sobre Desplazamiento Forzado en Colombia [citado 7 feb 2002]. Disponible en: www.col.ops-ms.org/desplazados/informes/rut/10/ default.htm\#enfermedades.

17. Summerfield D. War and mental health: a brief overview. BMJ 2000; 321(7255):232-235.

18. Ministerio de Salud - Instituto Nacional de Salud. Perfil Epidemiológico de la Población Desplazada en Barrio Mandela. Colombia [citado 5 feb 2002]. Disponible en: www.col.ops-oms.org/desplazados/informes/mandela/index.htm

19. Mollica R, Melnnes J, Sarajlic N, Lavelle J, Sarajlic I, Massagli M. Disability associated with psychiatric comorbidity and health status in Bosnian refugees Living in Croatia. JAMA 1999;282(5): 433-439.

20. Sánchez R, Jaramillo L. Desplazados experiencias en Salud, Impacto del Desplazamiento sobre la salud mental. Universitas Humanística. Bogotá 1999; XXVII (47): 87-101.

21. Asociación Probienestar de la Familia Colombiana Profamilia. Salud sexual y reproductiva en Zonas Marginales Situación de las Mujeres Desplazadas. Bogotá Profamila 2001.p.23-128

22. Castaño B. Experiencias en Rehabilitación y Recuperación Psicosocial. Libro Impacto de los conflictos armados en la Infancia. Memorias de la Consulta para la Región América Latina y del Caribe.
Convenio FES-UNICEF- Defensoría del Pueblo. Bogotá; 1996.p.44-49.

23. Consultoría para los Derechos Humanos y el Desplazamiento; Fondo de Las Naciones Unidas para la Infancia. Un país que Huye, Desplazamiento y Violencia en una Nación Fragmentada. Bogotá: Fondo de Las Naciones Unidas para la Infancia; 1999.

24. Carreño L, Millán H. Estudios diagnósticos para el fortalecimiento Integral de los proyectos de asistencia humanitaria de emergencia para personas internamente desplazadas. Chf-internacional. Corporación Avre [citado 21 Jul 2002]. Disponible en: www.disaster.info.desastres.net/desplazados/informes/avre/DiagnosticoFortalecimiento.doc.

25. Red de Solidaridad Social. Presidencia de la República. Sistema Nacional de Atención Integral a la Población Desplazada. Informe al Congreso de la República. Bogotá: Sistema Nacional de Atención Integral a la Población Desplazada;2002.

26. Conclusiones del I Encuentro de Responsables de Nutrición de Organizaciones de Ayuda Humanitaria en Colombia [citado 5 Feb 2002]. Disponible en: www.col.ops-oms.org/desplazados/documentos/ach/default.htm.

27. Meertens D. Víctimas y sobrevivientes de la guerra: Tres miradas de género. Libro Las Violencias inclusión creciente. Colección CES Bogotá: Universidad Nacional, Facultad de Ciencias Humanas; 1998; 236-65.

28. Ministerio de Salud, Organización Panamericana de la Salud. Lineamientos de atención psicosocial para la Población Desplazada por la Violencia en Colombia. Bogotá: Ministerio de Salud; 1999.

29. Goenjian AK, Steimberg AM, Najarian LM, et al Prospective study of posttraumatic stress, anxiety, and depresive reactions after earthquake and political violence. Am J Psychiatry. 2000; 157 (6): 911- 916. 